\title{
The Trajectory of Evolution and Its Implications for Humanity
}

\author{
John E. Stewart \\ Evolution, Complexity and Cognition Group \\ Center Leo Apostel for Interdisciplinary Studies \\ at the Vrije Universiteit Brussel in Belgium
}

\begin{abstract}
:
Does the Big History of life on Earth disclose a trajectory that has been driven by selection? If so, will the trajectory continue to apply into the future? This paper argues that such a trajectory exists, and examines some of its key implications. The most important consequence is that humanity can use the trajectory to guide how it evolves and adapts into the future. This is because the trajectory identifies a sequence of adaptations that will be favoured by selection. If humanity intentionally evolves its social systems and psychological capacities so that they follow the trajectory, humanity can avoid negative selection and instead survive and thrive indefinitely into the future. This would enable humanity to make a positive contribution to the future evolution of life in the universe. But it turns out that immediate selection will not drive the evolution of life on Earth further along this trajectory. Instead, intentional action by humanity is necessary. It is as if the evolution of life on any planet is a developmental process that has a very unusual characteristic: evolution will continue to develop successfully beyond a certain point only if it produces a sentient organism that: (i) awakens to the possibility it is embedded in a developing process; (ii) realizes that this developing process will continue successfully only if it chooses to intentionally drive the process forward; and (iii) commits to doing whatever is necessary to achieve this. On this planet, humanity is that sentient organism. The existence of such a key evolutionary role for humanity is capable of providing humanity with meaning and purpose in a larger scheme of things. For individuals who commit to driving the process forward, the nature of the trajectory has immediate consequences for what they should do with their lives, here and now.
\end{abstract}

Correspondence | John E. Stewart, future.evolution@gmail.com

Citation | Stewart, J. E. (2019) The Trajectory of Evolution and Its Implications for Humanity. Journal of Big History, III(3); 141 - 155.

DOI | https://doi.org/10.22339/jbh.v3i3.3380

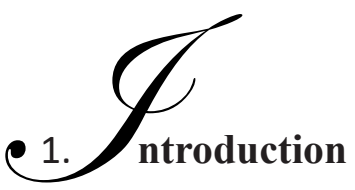

Until the emergence of humanity, evolution on Earth searched for adaptive improvements through the production of variation that was generated primarily by blind trial and error. Complex adaptations were discovered by the production of genetically variant offspring. These either flourished in competition with others, or more often were out-competed and became failed evolutionary experiments. The process which produced this exploratory variation had neither foresight nor any capacity to plan ahead. The process was not guided by any understanding of what might work in the future. As a consequence, it was a very wasteful process: in order to achieve a small improvement in any given adaptation, huge numbers of variant organisms were produced and failed. Nevertheless, given sufficient numbers of generations, complex adaptations could be discovered by this trialand-error searching of possibility space.

But with the emergence of humanity, all this changed. It took humans only a few thousand years to discover the means to fly through the air, while dinosaurs and birds took many millions. The development of two capacities in humans enabled this great leap forward in evolvability (the ability to discover effective adaptations). First, humans evolved the ability to construct mental models of how their environment would be impacted by their actions. They could then use these models to work out what particular actions would enable them to achieve their goals. No longer were adaptations discovered primarily 
by the actual production of variant offspring that were subject to selection. Instead, the initial production of variants and their selection was carried out mentally, within the heads of humans. This enabled humans to anticipate negative selection and adapt in ways that avoided it. The evolutionary process was internalized within the minds of humans. As the great evolutionary philosopher Karl Popper put it, this capacity "permits our hypotheses to die in our stead." (see Popper 1972; Dennett 1995)

The second capacity that enabled this significant enhancement of human evolvability was collective learning and other forms of cultural evolution (see Christian 2004; Boyd and Richerson 1985). This enabled mental models and other learning acquired by an individual to be passed on to others. Until this capacity emerged, what an individual learnt during its lifetime died with it. Each individual had to begin afresh to learn about its environment and how to manipulate it. But once such a capacity emerged, this learning could be passed on to others and could be transmitted across the generations as an accumulating culture of knowledge and skills. This could be used to generate better mental models of the environment and how it could be manipulated.

These two capabilities, powered by enhancements such as language and writing, progressively enabled humans to develop increasingly complex models of their past, present and future. This led to the emergence and growth of science and technology. Eventually it enabled the development of models of the evolution of life on Earth, including human evolution. A further significant development was the integration of various models across disciplines to produce Big History. These included models of the birth and evolution of the universe as well as life within it, up until the present.

However, humans have only just begun to use mental modelling for a purpose which is perhaps of greatest significance from a larger evolutionary perspective. This involves developing models of the possible evolutionary futures of humanity. These models could be used by humanity to identify actions and technologies that are essential for its future evolutionary success. Once humanity knows what characteristics are needed to avoid being selected against in the future, it can take the steps needed to adopt these characteristics.

Humanity may, for example, be able to identify a trajectory in evolution that extends into the future. Evolution exhibits a trajectory when one or more characteristics of organisms change in a particular direction as evolution unfolds. For example, there may be a progressive increase in the size or complexity of organisms or in the the scale of cooperative organization, or a population of organisms may evolve into a super-organism. Such a trajectory identifies a sequence of adaptive changes that would not be eliminated by selection and that would enable future survival. If such a trajectory exists, humanity could use its mental models to locate itself along the trajectory and to identify what actions it needs to take to align with the trajectory. Rather than having to be subject to actual selection in-the-world for evolution to proceed, humans could anticipate and avoid actual selection by using the less-destructive selection that occurs in mental and cultural processes. Aligning its development with evolution's trajectory in this way would enable humanity to adapt and survive indefinitely into the future. Humanity could avoid becoming a failed evolutionary experiment. Such a shift in which an organism begins to use the trajectory of evolution to guide its intentional adaptation and evolution constitutes a major evolutionary transition. I will refer to it as the transition to intentional evolution.

But is this possible? Does evolution have a trajectory that is driven by selection and that will continue into the future? Section 2 of this paper outlines the arguments and evidence that have been advanced in the past against the view that evolution has a driven trajectory. Section 3 demonstrates that these previous objections to directionality have been overcome by a relatively new hypothesis: that evolution embodies a driven trend towards increasing integration and 
cooperation. Section 4 outlines key implications of this trajectory for humanity. It demonstrates that given near-universal human goals, the trajectory has immediate consequences for what humans need to do to achieve their goals.

\section{The Case Against the Existence of a Driven Trajectory}

\subsection{The intentional exclusion of directionality from the Modern Evolutionary Synthesis}

Mainstream evolutionary biology takes the position that overall, evolution is not directional or progressive. In part, this position still prevails because of decisions made by the founders of what has become known as the Modern Evolutionary Synthesis (the body of theory and beliefs that came to underpin evolutionary science as it developed in the second half of the $20^{\text {th }}$ century). As outlined by evolutionary philosopher Michael Ruse in his book Monad to Man (Ruse 1996), in a series of meetings in the 1940s, the founders of the Synthesis set out to place the academic study of evolution on a firm scientific footing. With this goal in mind, they decided that ideas about direction and progress should be excluded from the discipline of evolution. As Ruse recounts, the main reason for this was profession building: the founders were concerned that controversies surrounding the implications of evolutionary directionality and progress would undermine their attempts to establish the study of evolution as a rigorous scientific discipline. These controversies included, for example, attempts to use evolutionary criteria to rank human races and cultures. Ruse outlines how the founders enforced their decision through their influence over the editorial policies of scientific journals and as respected peer reviewers of papers submitted for publication. Ironically as Ruse points out, the founders made this decision despite most of them considering that evolution is directional. Against this background, it is clear that the initial exclusion of directionality and progress from scientific studies of evolution did not result from any scientific case against these ideas.

\subsection{The absence of any 'impossibility proof'}

Furthermore, the case against directionality has never been buttressed by any kind of general 'impossibility proof' i.e. by any demonstration that the nature of the evolutionary process is such that overall directionality cannot emerge and has not done so. The nearest to such a proof has been the suggestion that natural selection only favours local adaptation to local conditions, and therefore cannot drive any overall advance across species (e.g. Gould 1996). But while it is true that natural selection generally favours only local adaptation, this obviously does not preclude the existence of adaptations that provide general fitness benefits as well as local benefits. This would include adaptations that tend to produce fitness advantages across many or all environments. For example, meta-adaptive adaptations such as improvements in evolvability can be advantageous in many environmental circumstances. So the local nature of adaptation does not itself preclude general improvements across species, or any overall trend or direction to evolution.

\subsection{The big poverty of Big Historicism}

Popper (1957, 1959) and more recently Taleb (2007) have mounted general arguments against the use of historical patterns and explanations to predict future trends and trajectories. Their arguments apply equally to the extrapolation of Big Historical and evolutionary patterns into the future. First they caution against the extrapolation of trajectories and other patterns in the absence of evidence that: (i) the patterns are causally driven rather than accidental emergences or other kinds of artefacts; and (ii) the causal micro-foundations will operate into the future, continuing to drive the pattern. They are right to argue that the elucidation of such causal micro-foundations is essential if any hypothetical extrapolated pattern is to be taken seriously. 
Second, they argue that historical 'just so' stories which can explain all relevant past events and all that might arise in the future, cannot provide reliable predictions of future trends. They are like the 'just so' stories that enable financial newspapers to explain everything that happened in the stock market yesterday, but are next to useless at predicting trends in tomorrow's market. Because they cannot make specific predictions, the hypotheses that are embedded in these historical stories are untestable and are not derived from testable theories. Their failure to be testable and falsifiable means that they should not be considered as scientific, according to Popper's widely accepted criteria for distinguishing science from nonscience.

These two fatal deficiencies bedevil attempts to extrapolate evolutionary and Big Historical trends into the future. To be taken seriously, any claim that a trend can be extrapolated into the future must satisfy these two criticisms. It must be accompanied by: (i) identification of the particular causal micro-foundations that drive the trend; and (ii) a demonstration that the claim is testable and falsifiable, and/or derived from hypotheses that are.

\subsection{A trend towards increasing complexity?}

Although the mainstream has continued to reject directionality, there is some support for the view that selection has driven an increase in the complexity of living processes as evolution unfolded (e.g. see Wilson 1992; Shanahan 2004; Christian 2004; Spier 2010; Vidal 2014). There is evidence for such a pattern: both the highest level of complexity and the average level of complexity of living processes have tended to increase progressively during the evolution of life on Earth. However, the hypothesis that these apparent patterns have been actually driven by selection has been heavily criticized for a number of reasons. In particular, it is clear that selection does not favour increases in complexity per se. There are many ways of becoming more complex that are not advantageous for any given organism, and selection often favours adaptations that decrease complexity. These difficulties are compounded by the fact that there is no agreed-upon definition of complexity that reflects intuitions about what it is that has increased (e.g. see Lineweaver et al. 2013).

Furthermore, Gould (1996) has demonstrated that even if selection did not drive increases in complexity, both the average and the highest complexity would be expected to increase in any event as evolution unfolds. The trend would arise passively, without being driven. This is because the evolution of life on Earth necessarily began with the simplest and least complex forms. The only way it could go from there was to explore more complex possibilities. Gould further pointed out that this drift to increasing complexity would not be countervailed by a drift towards decreasing complexity because life began with minimal complexity. Adopting his metaphor, life began near a 'left wall' of complexity.

Gould buttressed his argument against a driven trend toward increasing complexity with evidence that many lineages of organisms have not increased in complexity over many millions, and sometimes billions, of years. In particular he notes that bacteria have remained at the same level of complexity for billions of years and yet appear to be highly successful in evolutionary terms (they have survived and thrived, dominating life on Earth by mass and numbers). If there was an overall trend toward increasing complexity driven by selection, the complexity of bacteria and these other lineages would be expected to have increased significantly as evolution unfolded, Gould argues.

Proponents of a driven trend towards increasing complexity have not been able to counter these arguments by identifying the all-important causal micro-foundations that would drive such a trend. 


\section{The Mounting Case in Favour of a Trajectory towards Increasing Integration and Cooperation}

\subsection{An evident trend towards increasing integration}

Various versions of another large-scale pattern that is evident in the evolution of life on Earth has been identified by a number of evolutionary thinkers over the past century (e. g. see Teilhard de Chardin 1965; Corning 1983; Blitz 1992; Crawford 1992; Maynard Smith and Szathmáry 1995; Stewart 1995, 2000; Last 2017). This pattern resulted from a step-wise process in which living processes have been progressively integrated into organizations of increasing scale. It began with the integration of self-reproducing molecular processes into organizations that became the first simple cells; then organizations of these simple cells eventually formed the more complex eukaryote cell; this was followed by the integration of some of these cells into larger-scale organizations of cells, eventually emerging as multi-cellular organisms. In a further repetition of this process, organizations of multi-cellular organisms produced animal societies.

Importantly, this progressive integration of living processes has not been limited to evolution driven by gene-based natural selection. The trend has continued in human evolution where cultural evolutionary processes now predominate: small kin groups were integrated into bands, bands were integrated into tribes, these formed the constituents of kingdoms and city states, and these in turn have been integrated into nation states (Stewart 2000).

More abstractly, this step-wise process resulted from the integration of smaller-scale living entities into larger-scale entities as evolution proceeded. The larger-scale entities then repeated the process, becoming integrated into yet larger-scale entities. And so on, repeatedly. At each step, the larger-scale entities underwent an entification process, developing the capacity to evolve and adapt as coherent, organized individuals. Each step was also generally accompanied by increases in evolvability. Overall, the trajectory evident in the history of life on Earth has been toward the emergence of cooperative organizations of everincreasing scale, hierarchical depth and evolvability.

The result of this stepwise process of successive integrations is the nested hierarchical structure of living processes: if we look down into our bodies we see that we are a society of cells which in turn are organizations of the ancestors of simple cells which in turn are organizations of self-producing molecular processes. If we look above and beyond us we see that we are, for example, members of corporations and other organizations which are in turn members of nations, and these in turn are in some cases members of supra-national organizations such as the European Union.

\subsection{Is the trend towards increasing integration driven or passive?}

But has this apparent trajectory been driven by selection? A number of evolutionary researchers have argued that it has. They suggest that it has been driven by the potential advantages of cooperation between living entities (Corning 1983; Maynard Smith and Szathmáry 1995; Stewart 1995, 2000). These advantages result from the ability of cooperative organizations to take advantage of synergies, including those associated with division of labour and specialization. Furthermore, cooperatives are of larger scale than individuals and therefore can have greater command over resources, act effectively over larger scales, and have increased power and control over other living processes and their environment. These advantages enable effective cooperative organizations to out-compete isolated individuals in many situations. However, the hypothesis that the advantages of cooperation have driven evolution in the direction of increasing integration has been slow to attract mainstream support (Ruse 1996; Gould 1996; Shanahan 2004). First and foremost, this is because a central theme of mainstream evolutionary theory is that selfishness predominates in evolution, not cooperation (Williams 1966; Dawkins 1976). This is founded on the certainty that selfish individuals who take benefits produced 
by co-operators but who do not contribute anything in return will always tend to outcompete co-operators (selfish individuals include thieves, cheats and other free-riders). And altruistic co-operators who invest their resources in providing cooperative benefits to others but who do not receive cooperative benefits in return will always tend to be less fit.

A huge literature reports research which attempts to find special circumstances in which cooperation will predominate. But if anything, this research confirms that only in particular, constrained situations will cooperators be more competitive (for a brief overview see Stewart 2014). In general, this occurs only where special circumstances just happen to be present that guarantee that co-operators will capture sufficiently more of the benefits of cooperation than non-cooperators. The research has not identified a general mechanism that can account for the emergence of the complex cooperation found, for example, amongst cells in multicellular organisms, between molecular processes within a eukaryote cell, and amongst participants in modern human societies.

This widely-accepted view about the difficulties facing the emergence of complex cooperation appears to provide a strong case against the claim that selection drives evolution in the direction of increasing integration and cooperation. It is unlikely the claim will attract mainstream acceptance until it has been demonstrated that plausible evolutionary processes can drive the emergence of complex cooperation at all levels of organization, despite the evolutionary advantages that otherwise can accrue to free-riders and other non-co-operators.

\subsection{The causal micro-foundations of a trend towards increasing cooperation}

Arguably, this has been accomplished over the last two decades: Stewart $(1995,2000,2014)$ has shown that what he calls 'management' can enable the emergence of complex cooperative organization amongst self-interested entities that previously competed against each other. The management within an organization is comprised of processes that have the power to reward cooperative entities and to suppress free-riding entities. Within an organization that is managed effectively, it is therefore in the interests of entities to contribute cooperatively to the success of the organization as a whole, and against their interests to free ride. Useful cooperation pays, and the interests of members of the organization are aligned with the interests of the organization as a whole. 'Consequence-capture' will apply to all members of the organization: i.e. individuals will capture the benefits (or harms) produced by the impact of their actions on the organization (Stewart 2018). As a consequence, complex cooperation will tend to emerge and flourish where it benefits the organization.

Examples include: the management of proto-cells by RNA managers which support the production of cooperative enzymes and suppress free-riding side reactions; the management of a modern human corporation which remunerates cooperative employees who meet performance targets and fires free-riding employees who under-perform; and the government of a nation state which funds a defence force that cooperatively protects the nation, and punishes free-riding citizens who steal and break contractual obligations.

These three examples are instances in which management is external to the entities that are being managed. But management can also be internal to the entities and distributed across the organization. For example, management in early multicellular organisms and in insect societies is constituted by a cluster of genetic predispositions which are reproduced in each individual member across the organization. The predispositions can, for instance, predispose members to provide resources to co-operators. They can also organize the punishment of free riders. Distributed internal management is just as controlling and coercive as external, centralized management. However, the control exercised by distributed internal management is not readily visible. As a consequence, instances where it operates are often mistaken to be 
cases in which cooperative organization has somehow emerged spontaneously, in the absence of any form of control.

In general, the emergence of management is driven by its ability to promote cooperation within an organization and its capacity to capture sufficient of the extra benefits this produces. This tends to ensure a degree of 'consequence-capture' for management and therefore tends to align the interests of management with those of the organization as a whole. Selection operating at the level of the organization as a whole will tend to increase this coincidence of interests (e. g. see Wilson and Wilson 2007).

This theory of management supplies the piece that was missing from previous attempts to identify the causal micro-foundations that drive the trajectory of evolution towards increasing integration. It demonstrates how complex cooperative organization can emerge and flourish despite the forces that seem to favour selfishness and individualism. It identifies the causal mechanisms that enable isolated entities at one level to be organized into cooperatives that eventually become entities in their own right at the next level.

By providing comprehensive causal microfoundations, the supply of this missing piece also enables the trajectory to be validly extrapolated into the future. Broadly, it follows from the model that the step-wise process of integration into larger-scale organizations will repeat itself indefinitely. This is because, irrespective of the scale of entities that emerge at the highest level that exists at any point in evolution, there will be benefits that can be realized from cooperation between these entities of the largest scale (unless there is some absolute upper limit, such as the scale of a finite universe).

More specifically, extrapolation of the trajectory indicates that the next great step forward in the evolution of life on Earth would be the emergence of a living entity on the scale of the planet (Stewart 1995, 2000, 2014; Heylighen 2007). Initially, this would involve the management by human governance of a complex cooperative global organization which progressively integrates the planet's living processes (including human nation states and ecosystems), technology (including artificial intelligence), matter and energy (including the planet's biogeochemical cycles). Continuation of this trajectory would result in the global organization undergoing an entification process: the planetary organization would develop the capacity to establish its own goals and to pursue those goals by planning, acting and adapting as a coordinated and coherent whole. This would involve enhancing its own evolvability, including by modelling in more detail its own future possibilities.

The further extrapolation of the trajectory beyond this is straightforward: the human-managed entity would move out into space to establish new entities. As well as providing other adaptive advantages, this would enable the entity to avoid the extinction that would otherwise result from the engulfment of the Earth by the sun when the sun enters its Red Giant phase in a few billion years. Eventually, these new entities would link up with other planetary entities that originated elsewhere, forming cooperative organizations of yet larger scale and evolvability. Subject to any physical constraints that cannot be overcome, this would eventually result in the emergence of cooperative entities on the scale of galaxies and eventually the universe, infusing the universe with life and intelligence (including 'artificial' varieties). Each step in this future trajectory would again be driven by the advantages of cooperation over increasingly wider scales. Every global entity that emerges successfully and links up with others will bring unique contributions to the evolvability of the cooperative entities that it joins, due to its unique evolutionary history and unique perspectives.

\subsection{Testing the 'increasing integration' hypothesis against other objections.}

As we have seen, the major criticism levelled against previous claims that evolution is directional (including against earlier versions of the 'increasing integration' hypothesis) is that the claimed trends are not causally 
driven by selection. This left the claims open to the suggestion that they relied upon impermissible teleological explanations, including 'pulls from the future' (Ruse 1996; Shanahan 2004). However, the recent work outlined above has overcome this objection by identifying the causal micro-foundations that drive the trajectory towards increasing integration and evolvability.

This extended version of the case supporting the 'increasing integration' hypothesis also answers all the other key objections that have been made against previous attempts to demonstrate directionality (Stewart 2014). I will deal with each briefly in turn:

First and foremost, the mechanisms relied upon by the 'increasing integration' hypothesis are testable, as is the overall hypothesis itself. In particular, the key 'management' mechanism leads to clear predictions about the form of organization that will manifest in the cooperative organizations that arise at each level and become entities in their own right e.g. they will be organized as nested hierarchies, and each level will be organized initially by powerful management. This mechanism also lends itself to being tested effectively by appropriate simulations.

The 'increasing integration' hypothesis also makes clear predictions about how evolution will unfold on Earth in the short-term future. Destructive competition between human nations will tend to increase the possibility of nuclear war and environmental degradation resulting from global warming. The potential of international global cooperation to mitigate these and other threats will in turn tend to drive the emergence of international management in the form of global governance. By rewarding cooperative nations and suppressing freeriding nations, this governance would tend to align the interests of individuals, corporations, and nations with the interests of the global society. As discussed above, this would ultimately lead to the emergence of a cooperative living entity on the scale of the planet. However, there is no guarantee that the forces that tend to encourage these developments will succeed in overcoming the destructive competition that produces them, and human civilization might end this century.

The hypothesis also makes strong predictions about the forms of organization that will characterize living processes that emerge and evolve on other planets. Details will differ widely, but their forms of organization will unambiguously demonstrate that they have resulted from an evolutionary process characterized by the step-wise integration of living processes into cooperatives of increasing scale and depth.

The 'increasing integration' hypothesis also answers other objections that have been levelled against the 'increasing complexity' hypothesis. As mentioned above, proponents of the 'increasing complexity' hypotheses have been unable to develop an acceptable definition of complexity that matches intuitions about the nature of the complexity that appears to have increased. In contrast, the 'increasing integration' hypothesis resolves this difficulty by showing that only a particular form of complexity increases, and this form is clearly distinguishable from others (i.e. it is complexity resulting from the emergence of cooperative organization that increases overall, and this does not include, for example, the complexity of natural ecosystems because these are not managed cooperatives that evolve and act as coherent individuals).

As outlined above, the existence of lineages of organisms such as bacteria that have not increased in complexity for very long periods has counted against the 'increasing complexity' hypothesis. It can equally be argued that this also counts against the 'increasing integration' hypothesis because many lineages have not been integrated into large-scale cooperatives (yet). However, some species of bacteria have, in fact, been integrated into complex eukaryote cells which in turn have been integrated into multi-cellular organisms such as humanity (when humans go into space, organizations of the descendants of bacteria go with them). Bacteria have also played a critical role in scaffolding the emergence and evolution of life on 
Earth, including its potential to 'hatch' as a cooperative global entity.

Furthermore, the fact that some species of bacteria have failed so far to be integrated into larger-scale entities is not due to the absence of the cooperative advantages that are hypothesised to drive increasing integration. Rather it has been due to the absence of management arrangements that can cost/effectively reap the benefits of cooperation and drive further integration. For example, many bacteria live in spatially-restricted environments such as between soil particles. Such physically-restricted niches would not be able to be exploited by cooperatives of bacteria that are organized by management that can operate effectively only if the managed entities remain in physical contact with each other-the cooperatives would occupy too much space. This is why these niches have not been exploited by complex cells or multicellular organisms - they are organized by management that requires physical contact.

But the strongest reason to reject this objection in relation to the 'increasing integration' hypothesis is that these species that remained unintegrated are now increasingly being swept up into organizations managed by humans. This is expected to accelerate rapidly as a global organization emerges which incorporates an increasing proportion of the living and non-living processes of the planet, including biogeochemical cycles. In the long run, integration will tend to prevail as management emerges that is capable of exploiting the universal benefits of cooperation at all levels of organization, including at the global level (Stewart 2000, 2014; Lenton and Latour 2018).

For more detailed consideration of the evidence which supports the hypothesis that evolution proceeds in the direction of producing cooperative organization of increasing scale and evolvability, see Maynard Smith and Szathmary (1995) and Stewart (2000, 2014).

\section{Implications of the Trajectory for Humanity}

This model of the future trajectory of evolution can be used by humans to work out strategies for achieving their goals. The trajectory identifies the sequence of adaptations that humanity needs to make if it is to avoid becoming a casualty of selection. Humanity can locate itself along the trajectory and see how it must adapt if individuals and its societies are to survive and thrive into the future, and see what humanity must do if it is to contribute positively to the future evolution of life in the universe.

Before dealing in detail with the implications of this below, it is worth emphasizing the strength of the claim that is being made here. If humanity wants above all else to survive and thrive indefinitely into the future, it follows logically that humanity must align its development and evolution with the trajectory of evolution. This is as logically incontrovertible as the necessity for individual humans who want above all else to survive and thrive into the future to refrain from ingesting poisonous substances in quantities that will kill them quickly. If humanity wants to survive and thrive, there is no subjectivity involved. To ignore the trajectory or to pursue strategies that are not aligned with the trajectory is irrational. This strong claim will be substantiated in detail in the remainder of this section.

\subsection{Growing a cooperative and highly evolvable global entity}

Significantly, the consequences of the trajectory for humanity are not restricted to the far-off future. The next great steps in the evolution of life on Earth need to be taken in the near future if humanity and civilization is to ensure it survives this century. And the actions that individuals need to take to facilitate these steps need to have begun already.

In particular, the threats of nuclear war and pollution on the scale of the planet (e.g. global warming), necessitate the establishment of highly evolvable forms of global governance. These are needed to underpin a sustainable and cooperative global society that makes war between nations as unthinkable as war between the members of the United States of America 
and as unlikely as your liver attacking your brain.

Accordingly, individuals who make the transition to intentional evolution would work immediately for the establishment of global governance and a cooperative and unified global society. In order to further advance the evolutionary process along this trajectory, individuals would need to intentionally build the adaptive capability and evolvability of the global society. To achieve this, they would have to ensure that the global society undergoes the kind of entification process that cooperatives underwent at previous levels. They would need to establish processes and structures that enable it to set its own goals, develop mental models to guide what it must do to achieve its goals, make plans, and act as a coordinated and coherent whole. The global organization would need to be organized so that 'consequence-capture' applies to all entities within it, including those involved in establishing and adapting governance (Stewart 2018).

Importantly, in order to maximize its evolvability, the global system and its governance would need to be organized so as to minimize restrictions on the freedom and creativity of its members. Global governance would need to constrain citizens to the minimum extent necessary in order to align their interests with those of the global society. Citizens would then be free to pursue their aligned interests in whatever ways they choose. Furthermore, global governance would itself need to be constrained so that it cannot lead to exploitation, domination, or suppression of individual freedoms.

Enhancing the evolvability and creativity of all citizens would not be limited to the provision by governments of universal education. It would also extend to the universal provision of psychological support to facilitate the development by individuals of their psychological and cognitive capacities (Freinacht 2017). As was the result at all other levels when larger-scale cooperatives were organized by management, the establishment of effective global governance would massively increase diversity within the global society by enabling specialization, division of labour and other forms of cooperation that could not emerge otherwise. As occurred at lower levels, it would significantly increase the opportunities for individuals to engage in mutually-beneficial cooperative interactions, including through the suppression of destructive competition.

\subsection{Psychological and cognitive development}

Two particular psychological capacities would be critically important for enhancing the evolvability of the global society. Individuals who embrace intentional evolution will work on themselves to develop these capacities and promote their development in others.

The first is the ability to be psychologically selfevolving - the capacity for individuals to free themselves from the dictates of their biological and cultural past by being able to move at right angles to their existing emotional predispositions and motivations. Metaphorically, this includes the capacities 'to resist temptation' and 'to turn the other cheek', in their widest senses. This ability is important because our current motivations have been shaped by past evolution. As a consequence, they may clash with the motivations that are optimal for our future evolution. In order to be capable of doing whatever is necessary to meet the demands of future evolution, we need to be able to self-evolve so that we can find motivation and satisfaction in whatever that requires us to do (see Stewart 2001).

The second is the cognitive capacity to construct mental models of complex phenomena and to use these models to understand and manipulate complexity. Our current capacity for analytical/rational cognition (the 'formal operations' level of Piaget 1969) has proven very effective for modelling and understanding those aspects of the world that are relatively mechanistic and analysable. This capacity has driven the growth and spectacular success of science. But it is not effective for modelling and understanding more complex phenomena including social, economic, ecological and evolutionary systems. For this, what is known as meta-systemic thinking is needed. The development 
of this capacity will greatly enhance humanity's ability to deal effectively with all kinds of complex phenomena (see Stewart 2016).

\subsection{The critical importance of the transition to intentional evolution}

Significantly, the emergence and entification of a cooperative global organization cannot be expected to occur successfully unless humanity sets out intentionally to make it happen, guided by an understanding of the trajectory of evolution. This is because the processes that drove emergence and entification at lower levels of organization will not apply on Earth at the global level. At lower levels, competition between the members of a population of cooperatives drove selection that favoured those that were superior because they were more cooperative and evolvable. But at the global level there will obviously never be a population of global organizations that compete with each other. There can only be one global organization at a time. As a consequence, the emergence and entification of a global organization will not be driven by a competitive process operating between global organizations on Earth. Instead, the competitive process that drives the trajectory of evolution at the planetary level and beyond can only involve other global entities that originate elsewhere. The global entities that will survive and thrive in the universe will be those that anticipate and avoid the destructive selection arising from this inter-planetary competition and from astronomical events. Guided by an understanding of the trajectory of evolution, they will anticipate destructive selection by intentionally driving the entification process and linking up cooperatively with other entities that emerge elsewhere.

Global organizations that fail to anticipate this destructive selection will be in the same position as a member of a population of organisms that evolves blindly by trial-and-error processes. There is a very small probability that any given organism or its descendants will be the lineage that produces the particular sequence of mutations that constitute the next evolutionary step for the species. The overwhelming majority of lineages of organisms that evolve by blind trial and error will die out. The same would apply to the overwhelming majority of global organizations that adapt by blind trial-and-error. They are highly unlikely to stumble by accident on what they need to do to avoid destructive selection. The only way they can guarantee they will survive and thrive is to ensure that their ideas die in their stead. Evolving without being guided by the future trajectory of evolution is like driving a car by looking only in the rear-view mirror. A planetary civilization that evolves in this way is likely to be temporary.

Furthermore, other global entities that have successfully made this transition cannot be expected to intervene in the development of life on a planet that has not yet done so. If they were to make contact with the disparate living processes on a planet before a unified planetary organization has emerged, they would risk interfering with and undermining the successful development of a global entity. As they would know from their experience of making the transition, an effective emergence and entification process cannot be imposed externally. It must arise organically from within if it is to produce the complex internal structures and processes necessary for entification to proceed successfully. External interference would be like humans intervening in a chicken embryo and attempting to take over the manifold processes that produce the development and eventual hatching of the embryo.

External interference could also undermine the ability of an emerging entity to develop its own perspectives and capacities that would enable it to make unique contributions to larger-scale cooperatives it might link with. And it could be dangerous for the intervenors as well as life on the planet_living process that have not formed a planetary society will not have learnt the benefits of cooperation and how it can be organized successfully. For these reasons, life that has already emerged as a global entity and beyond is likely 
to have a strict non-interference policy in relation to planets at lower levels of development such as Earth. Understanding this resolves the Fermi paradox (for a detailed discussion of the paradox, see Webb 2015). However, once humanity grows a highly evolvable and cooperative global entity, life that has emerged elsewhere can be expected to appear (Stewart 2010).

In summary, a fully-developed global entity will emerge on Earth only if its development and entification is driven intentionally, by humanity. If humanity fails to do this, life on Earth will fail to hatch a global entity. Humanity and life on Earth will be a failed evolutionary experiment.

It is as if humanity is embedded in a developmental process that is directed at hatching a global entity. However, it is a very unusual developmental process. It will continue to unfold successfully only if humanity: (i) awakens to the possibility it is embedded in a developing process; (ii) realizes that this developing process will continue successfully only if humanity chooses to intentionally drive the process forward; and (iii) commits to doing whatever is necessary to achieve this (Stewart 2010).

Humanity is now at a stage in its evolution where it is faced with a fundamental existential choice. Will it make the transition to intentional evolution? Will it intentionally engineer a global entity that is capable of overcoming the threats that are currently faced by human civilization and that will arise in the future? Will humanity take the steps needed to ensure that Earth's global entity can link up with others to form a larger-scale entity that has the potential to exist indefinitely into the future?

Or alternatively, will humanity turn its back on life? Will it become irrelevant to the future evolution of life in the universe, denying life the unique capacities that humanity could contribute to larger-scale living organization? Will humanity choose to squat on the planet on which it emerged, pursuing the satisfaction of stone age desires and motivations that have been shaped by its past evolution, until its inevitable demise? Will humanity choose to ignore the trajectory of evolution, and instead continue modes of social organization and psychological functioning that will eventually be selected out of existence?

\subsection{Future-orientated evolutionary ethics and the naturalistic fallacy}

It can be strongly argued that a choice in favour of making the transition to intentional evolution would be required by human values that are near-universal. If humanity were to end without contributing positively to any on-going process, it would render meaningless and purposeless all human striving, history, sacrifice, science, art, and social and political progress. All positive human achievements will have come to nothing. It will be as if humanity and life on this planet had never existed. The near-universal drive to lead a life that is meaningful and that contributes positively to a scheme of things that is larger than oneself demands that humans keep humanity going. This is turn demands adapting and evolving along evolution's trajectory.

However, whether particular individuals awaken to the nature of this choice is dependent on their level of psychological and cognitive development. This was also the case, for example, in relation to the abolition of slavery. Sufficient support for the ending of slavery could not be attracted until significant numbers of citizens attained analytical/rational cognition with the emergence of the European Enlightenment. Attainment of this level of cognitive ability gave individuals the capacity to think and feel their way into the shoes of slaves who were living lives that they had never experienced themselves. However, analytical/rational cognition is not enough to enable individuals to think and feel their way into a full evolutionary worldview and its implications for humanity. Instead, metasystemic cognition is necessary for this, and few have achieved that capacity yet. However, the relatively recent emergence of Big History is an indication that the incidence of this capacity is increasing. Furthermore, the study of Big History can reasonably be expected to help develop the ability to build and 
operate mental models of large-scale systems as they evolve and interact.

Individuals who do make the transition to intentional evolution will use the evolutionary worldview to answer fundamental existential questions that face us all. What should I do? How should I live my life? Once they make the transition, the demands of future evolution will guide them as they decide what actions to take as their life unfolds. The injunctions and ethical principles that they follow will all be derived from their over-arching goal of positively serving the needs of future evolution. This goal leads to a completely different set of evolutionary ethics than one derivable from goals that succeeded in past evolution. Injunctions that led to evolutionary success in the past may lead to evolutionary disaster in the future.

Importantly, such a future-orientated evolutionary ethics does not run afoul of the naturalistic fallacy. This is because future-orientated ethics are not derived from facts alone. The fundamental drive to adopt these ethics comes from the desire to pursue near-universal human values. Unlike past-orientated evolutionary ethics, they are anchored in pre-exiting values and injunctions. And the naturalistic fallacy precludes deriving values from facts alone, not from existing values and facts. Individuals who take account of the longer-term evolutionary consequences of their actions when deciding how to act do not commit a philosophical fallacy, any more than do individuals who takes account of shorter-term consequences when deciding how to act (Stewart 2008).

In summary, there are many immediate actions that individuals who make the transition to intentional evolution would take to advance the evolutionary process on Earth and to enable human civilization to survive this century. Briefly, these include working for the emergence of a sustainable and unified global society, promoting in oneself and in others the development of enabling psychological capacities such as self-evolution and meta-systemic cognition, spreading the evolutionary worldview, working on the further development of the worldview, and so on (see
Stewart 2009 for more detail).

\section{Conclusion}

The trajectory of evolution sketched here can be expected to have unfolded elsewhere in the past and will unfold elsewhere in the future. This is because there is nothing in the nature of the causal microfoundations that have driven the trajectory on this planet that are unique to Earth. The same causal processes can be expected to drive the same step-wise increase in integration and evolvability wherever life emerges in the universe. The details will be different, but the forms of organization that constitute the trajectory can be expected to be similar. Management has emerged many times during the evolution of life on Earth, but the specific way in which it manifested has been different each time.

Wherever life with a capacity for mental modelling emerges in the universe, it can be expected to develop a science of evolution and its own Big History. Eventually it will develop models of the future evolution of itself and of the ecosystems in which it is embedded. Any such instance of intelligent life can be reasonably expected to discover the trajectory of evolution. This will enable it to realize that if the evolutionary process is to continue successfully in its case, there is a critically important role for it to play. It will come to understand that if it fulfils this role effectively, its unique history would enable it to contribute uniquely to any larger-scale cooperatives that it might join in the future. But any such instance of intelligent life will also know that if it fails to fulfil this role, it will constitute a failed evolutionary experiment. It will be faced with the same fundamental existential choice that faces humanity on this planet this century.

\section{Acknowledgements}

The author acknowledges useful discussions with Wilson Kennel and David Richards, and helpful comments on the manuscript from Ian Crawford. 


\section{References}

Blitz, D. 1992. Emergent Evolution: Qualitative Novelty and the Levels of Reality. Dordrecht: Kluwer Academic Publishers.

Boyd, R., and P. J. Richerson. 1985. Culture and the Evolutionary Process. Chicago: University of Chicago Press.

Christian, D. 2004. Maps of Time: An introduction to Big History. Berkeley: University of California Press.

Corning, P. A. 1983. The Synergism Hypothesis: A Theory of Progressive Evolution. New York: McGraw-Hill.

Crawford, I. A. 1992. "Beyond the Nation-State." Nature 358: 448. https://doi.org/10.1038/358448a0.

Dawkins, R. 1976. The Selfish Gene. New York: Oxford University Press.

Dennett, D. C. 1995. Darwin's Dangerous Idea. New York: Simon and Schuster.

Freinacht, H. 2017. The Listening Society: A Metamodern Guide to Politics, Book 1. Swiss Alps: Metamoderna ApS.

Gould, S. J. 1996. Full House: The Spread of Excellence from Plato to Darwin. New York: Harmony Books.

Heylighen, F. 2007. "The Global Superorganism: An Evolutionary-Cybernetic Model of the Emerging Network Society." Social Evolution and History 6: 58-119. http://pespmc1.vub.ac.be/Papers/ Superorganism.pdf.

Last, C. 2017. "Big Historical Foundations for Deep Future Speculations: Cosmic Evolution, Atechnogenesis, and Technocultural Civilization." Foundations of Science 22: 39-124. https://doi. org/10.1007/s10699-015-9434-y.

Lenton, T. M., and B. Latour. 2018. "Gaia 2.0." Science 361: 1066-68. https://doi.org/10.1126/ science.aau0427.
Lineweaver, C.H., Davies, P.C.W., and M. Ruse. 2013. "What is complexity? Is it increasing?" In Complexity and the Arrow of Time, edited by C. H. Lineweaver, P. C. W. Davies, and M. Ruse, 3-16. Cambridge, UK: Cambridge University Press.

Maynard Smith, J., and E. Szathmáry. 1995. The Major Transitions in Evolution. Oxford: Freeman.

Piaget, J. 1969. The Psychology of the Child. New York: Basic Books.

Popper, K. R. 1957. The Poverty of Historicism. Boston: Beacon Press

. 1959. The Logic of Scientific Discovery. London: Hutchinson.

1972. Objective Knowledge - An Evolutionary Approach. Oxford: Clarendon.

Ruse, M. 1996. Monad to Man: The Concept of Progress in Evolutionary Biology. Cambridge, MA: Harvard University Press.

Shanahan, T. 2004. The Evolution of Darwinism: Selection, Adaptation, and Progress in Evolutionary Biology. Cambridge, UK: Cambridge University Press.

Spier, F. 2010. Big History and the Future of Humanity. Chichester, UK: Wiley-Blackwell.

Stewart, J. E. 1995. "Metaevolution." Journal of Social and Evolutionary Systems 18: 113-114.

2000. Evolution's Arrow: The Direction of Evolution and the Future of Humanity. Canberra: Chapman Press.

—. 2001. "Future Psychological Evolution." Dynamical Psychology, http://www.goertzel.org/ dynapsyc/2001/PsyEv.htm.

- 2008. "The Evolutionary Manifesto." http:// www.evolutionarymanifesto.com/man.pdf.

2009. "Strategies for Advancing Evolution." http://www.evolutionarymanifesto.com/strategies. 
pdf.

. 2010. "The Meaning of Life in a Developing Universe." Foundations of Science 15: 395-409. https://doi.org/10.1007/s10699-010-9184-9.

2014. "The Direction of Evolution: The Rise of Cooperative Organization." Biosystems 123: 27-36. https://doi.org/10.1016/j.biosystems.2014.05.006.

. 2016. Review of Dialectical Thinking for Integral Leaders: A Primer, by Otto Laske. Integral Leadership Review, http://integralleadershipreview. com/14809-14809/.

- 2018. "Evolutionary Possibilities: Can a Society be Constrained so that 'The Good' SelfOrganizes?" World Futures 74: 1-35. https://doi.or $\mathrm{g} / 10.1080 / 02604027.2017 .1357985$

Taleb, N. N. 2007. The Black Swan: The Impact of the Highly Improbable. New York: Random House.

Teilhard de Chardin, P. 1965. The Phenomenon of Man. New York: Harper and Row.

Vidal, C. 2014. The Beginning and the End: The Meaning of Life in a Cosmological Perspective. New York: Springer.

Webb, S. 2015. If the Universe Is Teeming with Aliens ... Where is Everybody? Seventy-Five Solutions to the Fermi Paradox and the Problem of Extraterrestrial Life. 2nd ed. London: Springer.

Williams, G. C. 1966. Adaptation and Natural Selection. Princeton: Princeton University Press.

Wilson, D. S., and E. O. Wilson. 2007. "Rethinking the Foundation of Sociobiology." Quarterly Review of Biology 82: 327-348. http://dx.doi. org/10.1086/522809.

Wilson, E. O. 1992. The Diversity of Life. Cambridge, MA: Harvard University Press. 
